\title{
Quantification of antibody avidities and accurate detection of SARS-CoV-2 antibodies in serum and saliva on plasmonic substrates
}

\author{
Tiancheng Liu ${ }^{1,6}$, Jessica Hsiung, ${ }^{1,6}$, Su Zhao ${ }^{1,6}$, Jessica Kost ${ }^{1,6}$, Deepika Sreedhar', Carl V. Hanson², \\ Kjerstie Olson ${ }^{3}{ }^{3}$, Douglas Keare', Shin Ting Chang${ }^{1}$, Kevin P. Bliden ${ }^{4}$, Paul A. Gurbel ${ }^{4}$, \\ Udaya S. Tantry4, John Roche', Cynthia Press ${ }^{3}$, John Boggs ${ }^{3}$, Jorge P. Rodriguez-Soto ${ }^{3}$,

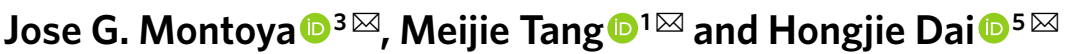

Accurate assays for the detection of antibodies to SARS-CoV-2 (severe acute respiratory syndrome coronavirus 2 ) are essential for the control of the COVID-19 (coronavirus disease 2019) pandemic. Here, we report antibody and antibody-avidity assays, relying on near-infrared-fluorescence amplification by nanostructured plasmonic gold substrates, for the simultaneous detection of antibodies to the S1 subunit of the spike protein and to the receptor binding domain of SARS-CoV-2 in human serum and saliva, and for quantifying immunoglobulin avidities against coronavirus antigens from SARS-CoV-2, SARS-CoV-1 and the common-cold viruses OC43, HKU1, NL63 and 229E. The antibody assay detected immunoglobulin M in 87\% (52 of 60 ) COVID-19-positive serum samples collected 6 or more days after symptom onset (and the immunoglobulins $M$ and $\mathbf{G}$ in all 33 samples collected at least 15 days after symptom onset), and correctly classified 456 out of the 457 COVID-19-negative serum samples tested (424 of them collected before the pandemic, including 73 that were positive for other viruses). We used the antibody-avidity assay to study antibody-maturation patterns, anamnestic responses, and cross-immunity to the common-cold coronaviruses.

S evere acute respiratory syndrome coronavirus 2 (SARS-CoV-2) has spread rapidly across the globe, causing millions of infections and over one million deaths within months. Currently, there is no effective vaccine or drug for preventing or treating coronavirus disease 2019 (COVID-19). The gold standard for COVID19 diagnosis is molecular detection of the SARS-CoV-2 virus by polymerase chain reaction with reverse transcription (RT-PCR) or isothermal nucleic acid amplification methods ${ }^{1-4}$. Antibody testing detects humoral immune responses to infection. It can be supplemental to a molecular test ${ }^{5,6}$, is more sensitive at later time points outside the viral ribonucleic acid (RNA) detection window, and can be performed in resource-limited regions. Developing antibody tests is also critical to surveillance and prevalence studies, assessing the antibody levels and duration needed for immunity, and evaluating vaccine efficacy. So far, various antibody tests have been developed using lateral-flow, enzyme-linked immunosorbent assay (ELISA) and chemiluminescence (CLIA) technologies ${ }^{6-15}$, but few differentiate antibody subtypes with $>99.5 \%$ specificity for both immunoglobulin-G (IgG) and $\operatorname{IgM}^{16,17}$. Highly specific antibody tests are critical to avoid misinterpretations or false positives, unnecessary stress and quarantine, and to prevent controversies and wrong conclusions for surveillance or prevalence studies ${ }^{17-23}$. On the other hand, no antibody-avidity test ${ }^{24-30}$ currently exists for COVID-19 to assess antibody maturation and distinguish recent versus old infections. Also, no SARS-CoV-2 antibody test currently offers testing of non-invasive matrices such as saliva, which could facilitate population-based mass screening of COVID-19. Finally, it is of fundamental importance to understand antibody responses against different antigens, cross-reactivity to other human coronaviruses, antibody maturation over time, and the roles these factors play in immunity.

With the rapid advances of nanotechnology, a nanostructured plasmonic gold (pGOLD) substrate ${ }^{29,31-34}$ has been developed for highly sensitive serology antibody assays. The pGOLD substrate is composed of nanoscale gold islands with abundant nanogaps, affording near-infrared (NIR) fluorescence enhancement by up to $~ 100$-fold due to plasmonic resonance and local electric field enhancements ${ }^{29,31-34}$. The increased NIR signal-to-background ratio on pGOLD allows multiplexed detection of panels of biological analytes over wide dynamic ranges. Previous antigen arrays on PGOLD have simultaneously detected IgG, IgM and IgA antibody subtypes for type 1 diabetes $^{32}$, toxoplasmosis ${ }^{34}$, hepatitis delta virus (HDV) ${ }^{35}$, and Zika and Dengue viral infections ${ }^{29}$. In particular, the pGOLD toxoplasmosis ${ }^{34}$ and $\mathrm{HDV}^{35}$ IgG antibody assays reached $\sim 100 \%$ sensitivity because of the exquisite NIR detection capabilities on the nanotechnology substrate. Here, we report the development of a high-performance, semi-quantitative assay for the detection of IgG, IgM and IgG avidity against the S1 subunit of the spike protein and against the receptor binding domain (RBD) $)^{5,36,37}$ of SARS-CoV-2 and other coronaviruses on the pGOLD substrate, in human serum and saliva.

'Nirmidas Biotech Inc., Palo Alto, CA, USA. ${ }^{2}$ California Department of Public Health Viral and Rickettsia Disease Laboratory, Richmond, CA, USA. ${ }^{3}$ Dr Jack S. Remington Laboratory for Specialty Diagnostics, National Reference Center for the Study and Diagnosis of Toxoplasmosis, Palo Alto Medical Foundation, Palo Alto, CA, USA. 'Sinai Center for Thrombosis Research and Drug Development, Sinai Hospital of Baltimore, Lifebridge Health, Baltimore, MD, USA. ${ }^{5}$ Department of Chemistry and Bio-X, Stanford University, Stanford, CA, USA. ${ }^{6}$ These authors contributed equally: Tiancheng Liu, Jessica Hsiung, Su Zhao, Jessica Kost.凶e-mail: montoyj@sutterhealth.org; meijie.tang@nirmidas.com; hdai@stanford.edu 
a

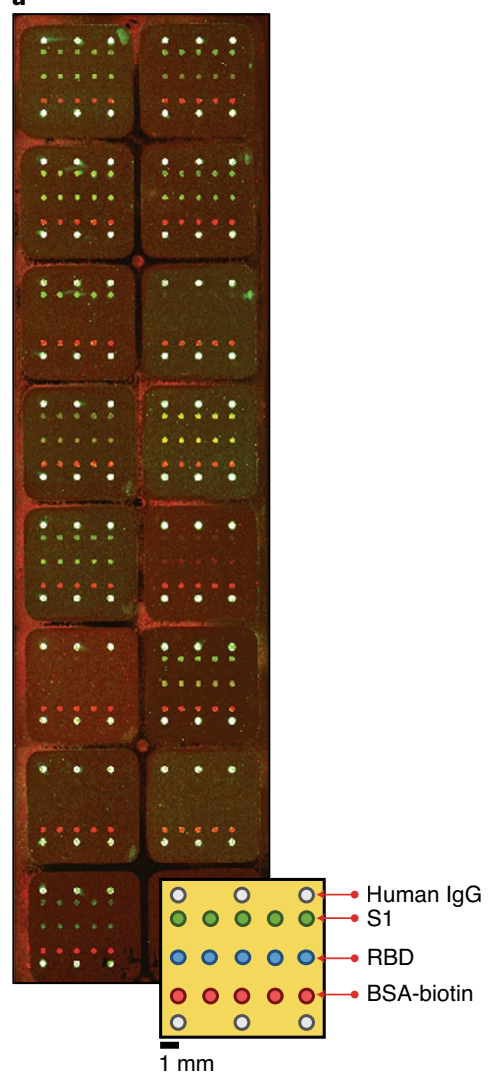

b
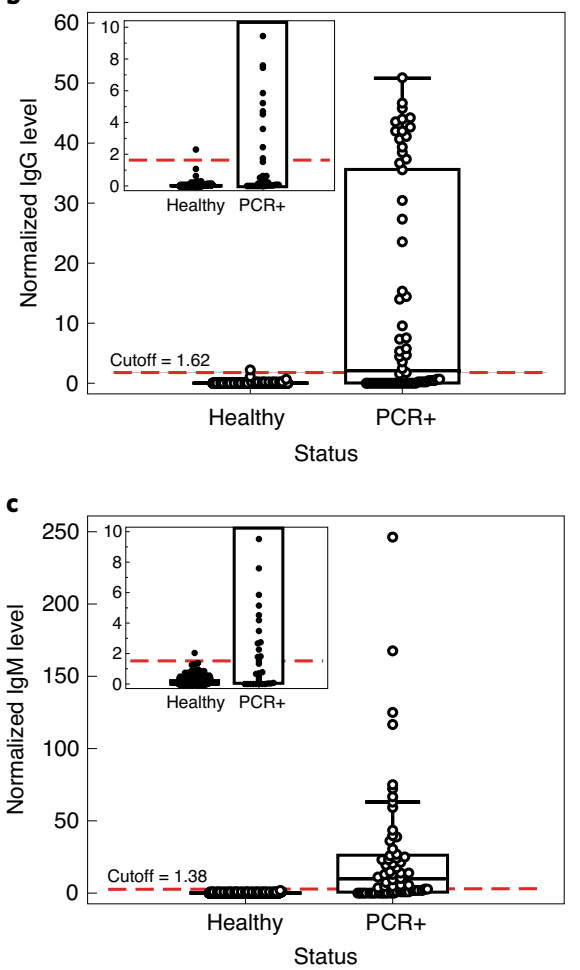

d

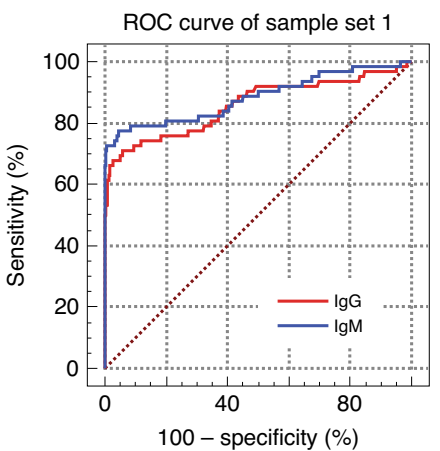

ROC curve of sample set 2

e

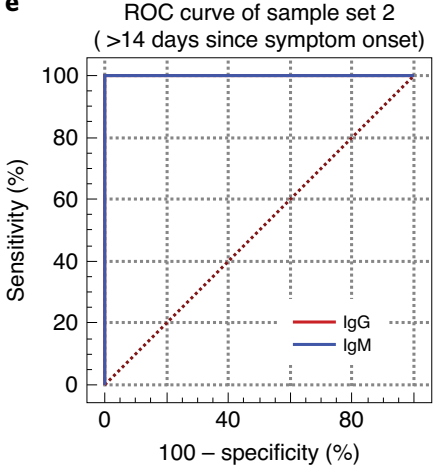

Fig. 1 | A nanoplasmonic assay for SARS-CoV-2 antibody testing. a, An overlay of confocal fluorescence scanned images of lgG (green) and lgM (red) channels acquired after testing serum samples in isolated wells (square-shaped regions). Yellowish-green coloured spots correspond to the detection of both IgG and IgM in the sample. The lower right schematic shows the printing layout of S1 (in green) and RBD (in blue) antigens and human IgG control spots (in white) in each well. The BSA-biotin spots (in red) are always labelled by a streptavidin dye to be detected in the lgM fluorescence channel and serve as an intrawell signal normalizer. b, IgG levels against S1 detected in COVID-19 PCR-negative or pre-pandemic presumptive negative ('Healthy', $n=384$ ) and COVID-19 PCR-positive ('PCR+', $n=62$ ) samples. The IgG level is defined as IgG channel fluorescence MFI divided by intrawell normalizer signal multiplied by 10. An IgG-level cutoff value of 1.62 is indicated as a red dashed line. Inset: a close-up of normalized IgG levels of healthy and PCR+ samples near the cutoff, with one false positive. $\mathbf{c}$, The same as $\mathbf{b}$, but for IgM levels. The lgM level is defined as IgM channel fluorescence MFI divided by intrawell normalizer signal multiplied by 100 , with a determined cutoff value of 1.38 (red dashed line). Inset: a close-up of IgM levels of healthy and PCR+ samples near the cutoff, with one false positive. d, ROC curve for the pGOLD SARS-CoV-2 IgG/lgM assay based on 384 negative and 62 PCR-positive COVID-19 sera ('sample set 1'; collected from patients with PCR-confirmed COVID-19, without symptom onset date information), which were used to establish IgG and IgM cutoffs. e, ROC curve for the pGOLD SARS-CoV-2 IgG/IgM assay based on 384 negative and 33 PCR-positive COVID-19 serum samples collected 15 to 45 days post symptom onset (a subset of 'sample set 2'). Data in $\mathbf{b}$ and $\mathbf{c}$ are presented as box plots with the minima, maxima, median line, interquartile range, data points and outliers shown.

\section{Results}

Development of the pGOLD SARS-CoV-2 IgG/IgM assay. We first fabricated and optimized arrays of SARS-CoV-2 spike protein $\mathrm{S} 1$ subunit and $\mathrm{RBD}$ antigen spots on a pGOLD slide in a microarray format (Fig. 1a) for capturing IgG and IgM antibodies in a sample, followed by labelling of the captured antibodies with anti-human IgG-IRDye800 and anti-human IgM-CF647 dye (Methods). The pGOLD biochip was then imaged by a confocal microscopy scanner in the red and NIR channels. The IgG and IgM antibodies bound to each antigen spot (Fig. 1a) were analysed through the fluorescence intensities of IRDye800 and CF647 dye, respectively. Dilution of a pure humanized SARS-CoV-2 IgG antibody solution over four orders of magnitude led to signal changes by $\sim 4$ logs, giving an estimated antibody detection limit of $\sim 1.6 \mathrm{ng} \mathrm{ml}^{-1}$ (Supplementary Figs. 1 and 2). A PCR-confirmed COVID-19-positive sample was diluted by up to $10^{5}$ times, and antibody signals were still well above the background noise across all dilutions (Supplementary Figs. 1 and 2). These results suggest the high analytical sensitivity and wide dynamic range of the multiplexed pGOLD assay.

Performance of the pGOLD SARS-CoV-2 S1 IgG/IgM assay. We detected SARS-CoV-2 antibodies on the pGOLD assay in human serum samples, first focusing on antibodies against the S1 antigen (Fig. 1). To determine specificity, we tested a total of 384 negative and presumptive negative samples, including 33 from PCR-confirmed COVID-19-negative individuals, 311 pre-pandemic samples (collected in 2017-2019) and 40 healthy control samples acquired before the COVID-19 outbreak. We also obtained a set of sera from 62 PCR-confirmed COVID-19 patients (without information given regarding the number of days between symptom onset to sample collection, referred to as 'sample set 1'). Receiver operating characteristics (ROC) curve analysis was performed based on the quantification of normalized antibody levels from median fluorescence intensity (MFI) signal outputs for the 384 negative and 62 positive samples (Fig. 1d). The cutoff values were determined under the 


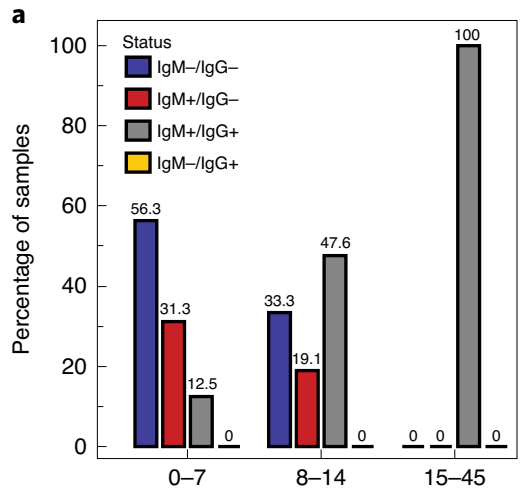

Days since symptom onset
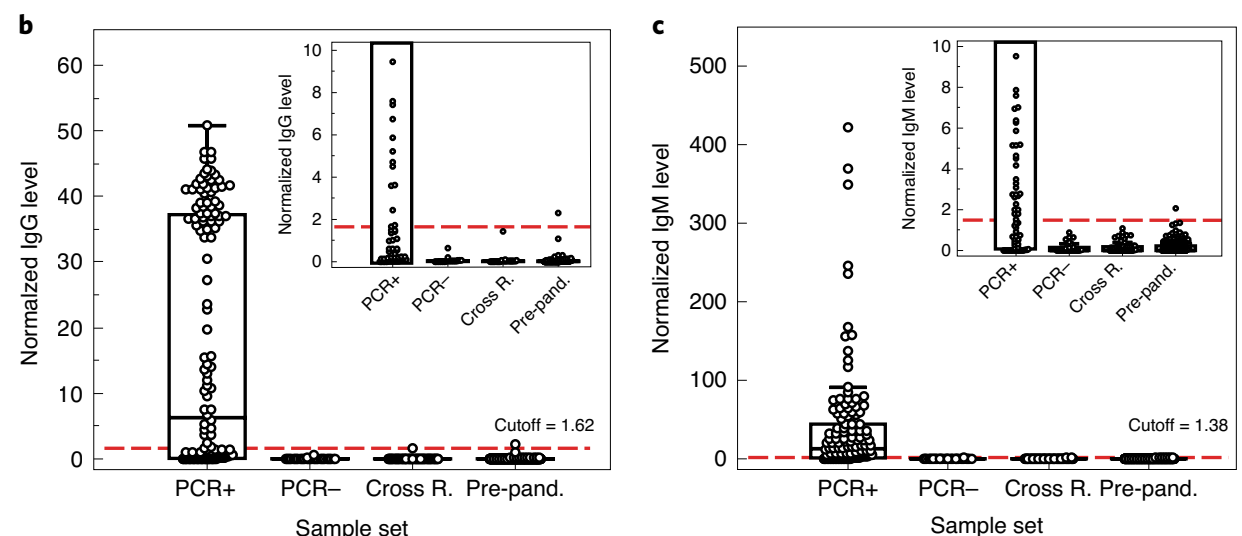

Fig. 2 | Highly sensitive and specific SARS-CoV-2 antibody test. a, Percentage of samples with IgM/lgG antibody status combinations according to days from symptom onset to sample collection date in the ranges 0-7, 8-14 and 15-45 days. Data based on 'sample set 2' are from patients with COVID-19, collected on specific days post symptom onset. b. Box plots of IgG levels detected in four sets of serum samples against the $\$ 1$ antigen with a cutoff value of 1.62 displayed as a red dashed line. Inset: a close-up of normalized IgG levels by sample set near the cutoff with one false positive. 'PCR+' denotes serum samples from patients who tested positive by PCR for COVID-19 $(n=136)$ and 'PCR-' denotes those who tested negative ( $n=33$ ). 'Pre-pand.' corresponds to pre-pandemic collected samples $(n=351)$. 'Cross R.' corresponds to samples from patients with other diseases for cross-reactivity evaluation ( $n=73$ ). c, The same as $\mathbf{b}$, except for lgM levels, with a cutoff value of 1.38. Inset: a close-up of normalized lgM levels by sample set near the cutoff, with one false positive. Data in $\mathbf{b}$ and $\mathbf{c}$ are presented as box plots with the minima, maxima, median line, interquartile range, data points and outliers shown.

criteria of $>99.5 \%$ specificity while maximizing the sensitivity for detecting both IgG and IgM in the sera of COVID-19 patients (Fig. $1 b, c)$. Under this condition, only one serum sample from the 384 presumptive negative set was found to be false positive.

To further establish pGOLD assay specificity and potential cross-reactivity, we tested 73 pre-pandemic samples collected from patients with various diseases, including influenza, Mycoplasma pneumoniae, autoimmune disease, hepatitis B virus (HBV), hepatitis $\mathrm{C}$ virus (HCV) and human immunodeficiency virus (HIV) (Supplementary Table 1). All of these samples were found negative for IgG and IgM against SARS-CoV-2 based on the established cutoff values, suggesting near zero cross-reactivity of the pGOLD assay (Fig. 2b,c). Moreover, antibodies from patients infected with common-cold strains 229E, NL63, HKU1 and OC43 did not cross-react with SARS-CoV-2 S1 and RBD antigens on the pGOLD assay. Altogether, with a total of 457 pre-pandemic presumptive negative and PCR-confirmed negative samples (Fig. 2b,c and Supplementary Table 2), only one sample was false positive in IgG and IgM, resulting in an overall specificity of $99.78 \%$ for both antibody isotypes.

Post symptom onset related positive rates of IgG/IgM against $\mathrm{S1}$. For the 62 PCR-confirmed COVID-19 samples collected without days since symptom onset data, the sensitivity of the pGOLD assay was $51.61 \%$ for IgG and $70.97 \%$ for IgM (Fig. 1d), suggesting a substantial fraction of each sample was collected in the early stage $(\leq 14$ days) of SARS-CoV-2 infection, before the development of antibodies $^{6-13,15,38}$. To investigate the immune response and sensitivity of the pGOLD SARS-CoV-2 antibody assay at various times post infection, we measured an independent set of sera from 70 patients with PCR-confirmed COVID-19 collected between 0 and 45 days post symptom onset ('sample set 2'). Assessment of antibody status in the second set of samples was performed using the cutoffs generated with the first PCR-positive sample set. Based on the days from symptom onset, PCR-positive patients were divided into three groups: I (0-7 days), II (8-14 days) and III (>14 days). We found that the positive rates for IgM in each group (I, II and III) were $43.75 \%, 66.67 \%$ and $100 \%$, respectively (Supplementary Table 3). The positive rates for IgG in each group (I, II and III) were $12.5 \%$,
$47.62 \%$ and $100 \%$, respectively (Supplementary Table 3 ). The results indicate a higher positivity rate of IgM than IgG initially (Group I) and $\sim 100 \%$ for both IgG and IgM antibodies in all patients at a later stage post infection (Group III).

In Group I (0-7 days post infection), $\sim 56.25 \%$ of patients were negative for both IgM and IgG, 31.25\% of patients developed IgM but not $\mathrm{IgG}$, and $12.5 \%$ of patients developed $\operatorname{IgM}$ and $\mathrm{IgG}$, clearly showing the presence of IgM preceding IgG as the initial immune response against COVID-19 infection (Fig. 2a). In Group II, 47.62\% of patients developed both IgM and IgG, compared to $19.05 \%$ of patients who were positive for IgM and negative for IgG within the same onset group. By $>14$ days post infection, $100 \%$ of patients had developed both IgG and IgM against SARS-CoV-2 (Fig. 2a). Combined, the antibody positive rates for $\geq 6$ days, $\geq 10$ days and $>14$ (15-45) days were $75 \%$ for IgG and $86.67 \%$ for $\operatorname{IgM}, 87.76 \%$ for IgG and $93.87 \%$ for IgM, and $100 \%$ for IgG and $100 \%$ for IgM, respectively (Supplementary Table 4 ). The pGOLD assay attained $100 \%$ sensitivity for sera from patients with COVID-19 collected $>14$ days post infection and $99.78 \%$ specificity in detecting both IgG and IgM subtypes against the $\mathrm{S} 1$ antigen overall (Fig. 1e).

Correlation analysis of two-plex antibody detection against S1 and RBS. To investigate antibody responses to specific regions of the SARS-CoV-2 spike protein, we measured and analysed antibodies against RBD in the $S 1$ subunit simultaneously on the positive and negative sample sets. We obtained a maximum specificity of $99.78 \%$ for IgG (1/457 false positive, the same anti-S1 IgG false positive) and $99.78 \%$ for IgM (1/457 false positive, a different sample from the anti-S1 IgM false positive) and a sensitivity of $100 \%$ for both IgG and IgM in PCR-confirmed COVID-19 samples at $>14$ days post symptom onset, suggesting that the pGOLD assay of antibodies against RBD was also highly specific and sensitive (Supplementary Tables 5 and 6).

Correlation analysis of antibodies in COVID-19 sera against S1 and RBD deviated from linear relations, with IgG levels against the two antigens scattered around the slope $=1$ line (Fig. 3a). The IgM levels against $\mathrm{S} 1$ and $\mathrm{RBD}$ were scattered with a fit-line slope well below 1, suggesting substantially higher IgM binding to RBD than to $\mathrm{S} 1$ (Fig. 3b). The RBD in the S1 subunit in the SARS-CoV-2 spike 

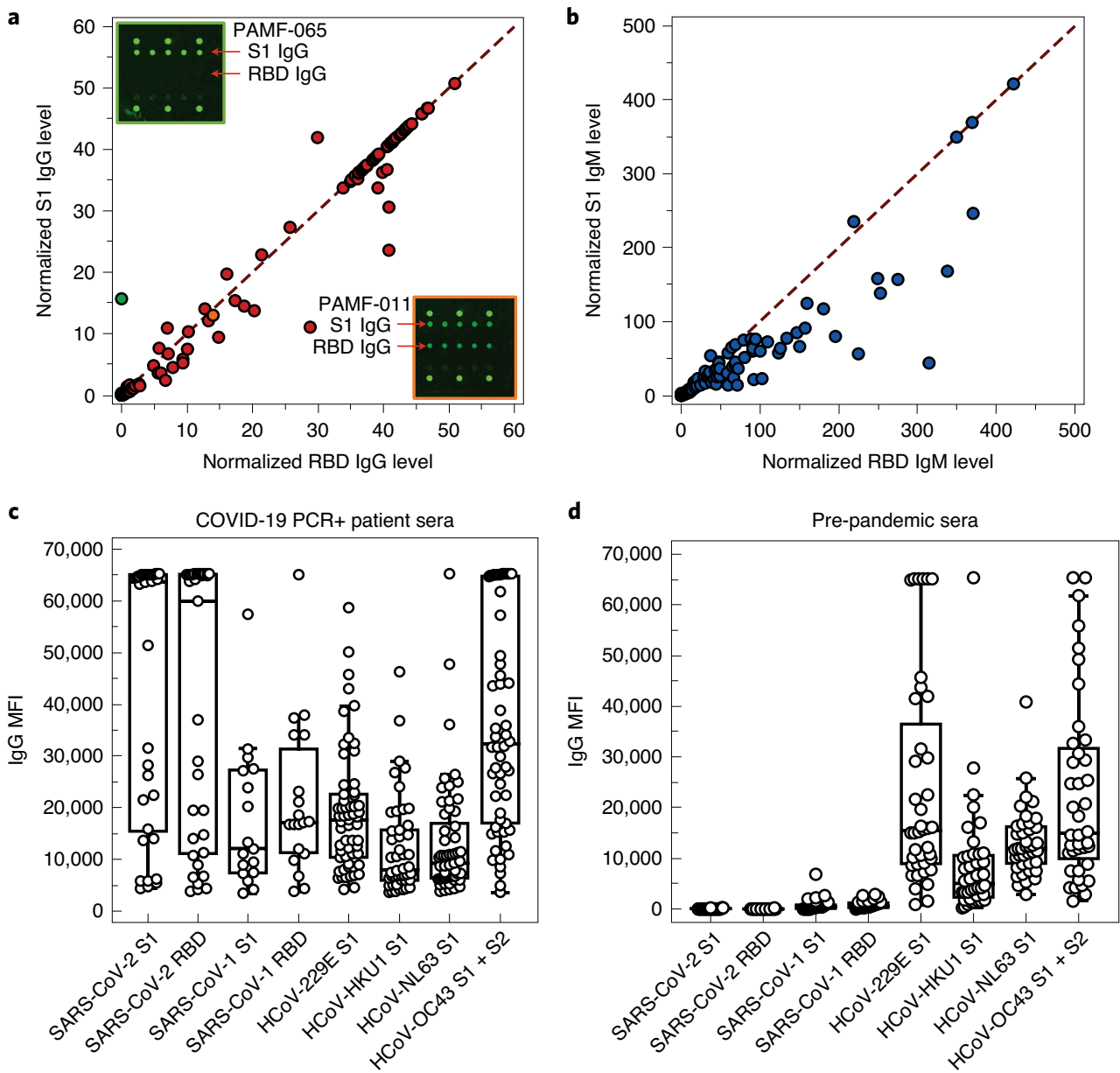

Fig. 3 Multiplexed antibody assay against various antigens of novel and previous coronaviruses. a, Correlation plot of normalized anti-S1 lgG level ( $y$ axis) and normalized anti-RBD IgG level ( $x$ axis) measured in PCR-confirmed COVID-19 sera $(n=136)$. The dashed line is drawn to have a slope of 1 . The upper left inset (outlined in green) shows a scanned image of the lgG-only channel in a patient serum labelled as PAMF-065, which displays high signal on the $\mathrm{S} 1$ antigen but not on the RBD antigen. The lower right inset (outlined in orange) shows a scanned image of lgG levels of a sample labelled as PAMF011, displaying approximately equal lgG signals against S1 and RBD. b, Correlation plot of normalized anti-S1 lgM level ( $y$ axis) and normalized anti-RBD IgM level ( $x$ axis) measured in COVID-19 PCR-positive sera $(n=136)$. The dashed line is drawn to have a slope of 1. c, MFI of IgG detected in COVID-19 PCR-positive sera $(n=62)$ against S1 of SARS-CoV-2, SARS-CoV-1 and four common human coronaviruses (HCoV) 229E, HKU1, NL63 and OC43, and against RBD of SARS-CoV-2 and SARS-CoV- 1 only. $\mathbf{d}$, The same as $\mathbf{c}$, but for pre-pandemic sera $(n=40)$. The raw data corresponding to $\mathbf{a}$ and $\mathbf{b}$ are provided in Supplementary Table 7. Data in $\mathbf{c}$ and $\mathbf{d}$ are presented as box plots with the minima, maxima, median line, interquartile range, data points and outliers shown.

protein is responsible for binding to the angiotensin-converting enzyme 2 (ACE2) receptor on host cells to initiate infection ${ }^{36,39}$, and is generally recognized as an important target for both neutralization antibody treatment and specific SARS-CoV-2 detection. Our results showed that the S1 and RBD antigens were both highly specific and sensitive SARS-CoV-2 antibody targets, while complementing each other in sensitivity to a discernible degree (Supplementary Table 7). To exploit the two-plex capability, we were able to combine antibodies against $\mathrm{S} 1$ and RBD to increase the $\mathrm{pGOLD}$ assay sensitivity for the $\leq 14$-day sample group for IgG and IgM, from $32.43 \%$ against S1 only to $37.84 \%$ combined and $54.05 \%$ against RBD only to $56.76 \%$ combined, respectively.

Intriguingly, an extreme case was that serum from one patient with COVID-19 (labelled as PAMF-065) showed SARS-CoV-2 IgG only binding to $\mathrm{S} 1$ and not to RBD (Fig. 3a, green inset). A recent study has identified distinct groups of phage-display-derived antibodies against SARS-CoV-2 that bind preferentially to RBD or $\mathrm{S1}^{40}$. Our results could reflect a similar phenomenon in human COVID-
19 serum, but requires further understanding of the immune responses to SARS-CoV-2 and the antibody-antigen interactions at the molecular level ${ }^{41}$.

SARS-CoV-2 IgG avidity test. A SARS-CoV-2 IgG avidity assay was developed on pGOLD to investigate antibody-antigen binding affinity and stability in denaturing conditions $s^{25-30}$. In our pGOLD SARS-CoV-2 IgG avidity test, a denaturing $6 \mathrm{M}$ urea treatment step was introduced to remove weakly bound antibodies on the antigen, leaving only the antibodies with a strong affinity for the antigens on pGOLD to be detected. We found that IgG against S1 and RBD in all COVID-19 sera except one (PAMF-065) showed a low avidity between 0 and 0.3 (Fig. 4a,b and Supplementary Table 8), consistent with recent infections, since all of our IgG-positive COVID-19 samples were collected within 6 and 45 days post infection. A slight trend of higher anti-S1 IgG avidity versus the number of days post symptom onset was discerned (Fig. 4a). Also noticeable was a lower average anti-RBD IgG avidity than anti-S1 IgG avidity. 
a

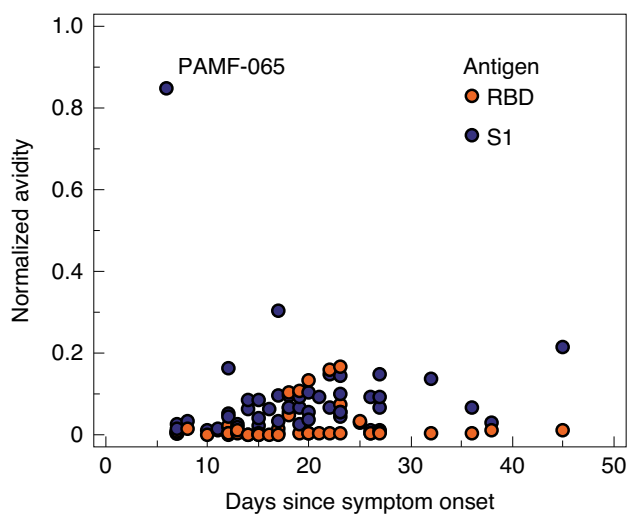

c

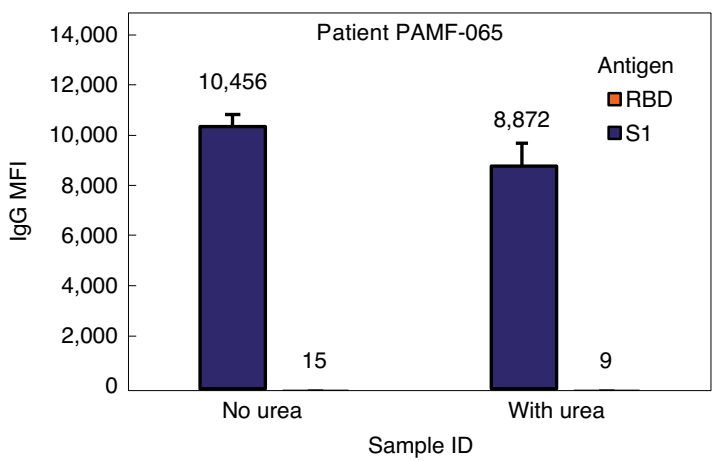

b

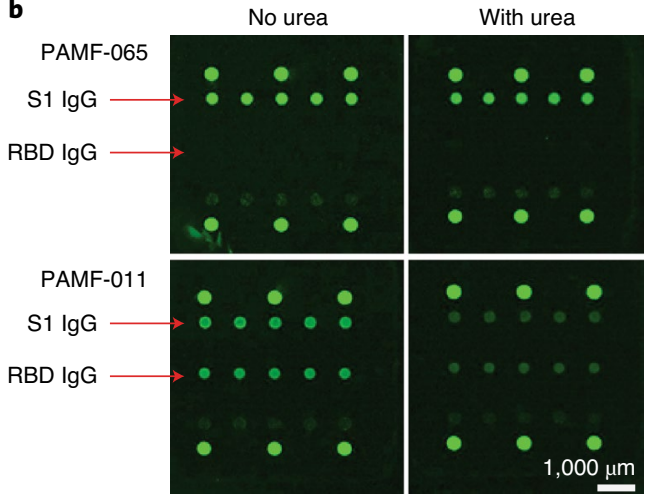

d

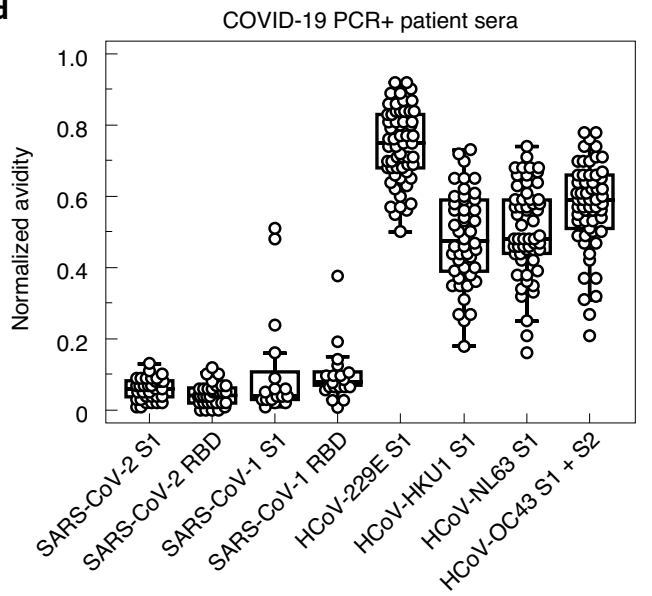

Fig. 4 | Antibody avidity against novel and previous coronaviruses. a, Normalized avidity of anti-S1 IgG and anti-RBD IgG measured in IgG-positive, PCR-confirmed COVID-19 sera $(n=49)$ collected 6 to 45 days post symptom onset. Avidity was calculated as the MFI of the urea-treated sample divided by the MFI of the non-treated sample. The serum of PAMF-065 showed unusually high avidity for anti-S1 IgG while being negative for anti-RBD IgG. b, Upper panel: fluorescence images of the IgG-only channel, showing the PAMF-065 serum sample with high anti-S1 IgG level with and without urea treatment, hence high avidity. It showed negligible anti-RBD IgG. Lower panel: fluorescence images showing another patient serum tested, PAMF-011, with much reduced anti-S1 IgG level after urea treatment, indicating low avidity. Low avidity was observed for all samples except PAMF-065. c, Anti-S1 IgG MFI signals of the PAMF-065 sample with and without urea treatment. Error bars indicate one standard deviation from the mean of the MFI signals detected against the respective microarrayed antigen spots $(n=3)$. d, Normalized avidity measured in COVID-19 PCR-positive and IgG-positive sera ( $n=62$ ) against S1 of SARS-CoV-2, SARS-CoV-1 and four common human coronaviruses (HCoV) 229E, HKU1, NL63 and OC43, and against RBD of SARS-CoV-2 and SARS-CoV-1 only. The raw data corresponding to $\mathbf{a}$ and $\mathbf{c}$ are provided in Supplementary Table 8 . Data in $\mathbf{d}$ are presented as box plots with the minima, maxima, median line, interquartile range, data points and outliers shown.

Surprisingly, sample PAMF-065 (with antibody binding only to S1 and not to RBD, Fig. 4b upper images), collected from a patient with PCR-confirmed COVID-19, showed a strong anomaly in anti-S1 IgG avidity, with a high value of $\sim 0.8$ (Fig. 4 c), typically interpreted as an infection from more than six months previously. The serum sample used for the pGOLD antibody assay, which was collected from the patient six days post symptom onset, showed a high anti-S1 IgG level at 10 times the cutoff, but a low anti-S1 IgM level at $\sim 1.4$ times the cutoff. The unusually rapid rise in IgG resembles the response of acute Zika-infected individuals who had old Dengue infection $^{29,42}$. Based on PAMF-065 showing an anamnestic response of high IgG against SARS-CoV-2 S1 at six days post symptom onset, low positive IgM and unusually high IgG avidity, we infer that the patient was previously exposed to a closely related infection including other coronaviruses with antibodies that cross-react with SARS-CoV-2 $\mathrm{S}^{43}$ but not SARS-CoV-2 RBD. This case is intriguing and underscores the usefulness and importance of antibody-avidity testing for COVID-19.
Multiplexed pGOLD antibody and antibody-avidity assay for human coronaviruses. We fabricated pGOLD biochips with eight antigens in an array, including SARS-CoV-2 S1 and RBD, SARS-CoV-1 (SARS for short) S1 and RBD, and four common-cold coronaviruses OC43, HKU1, NL63 and 229E S1 antigens and performed multiplexed antibody and antibody-avidity assays. With or without COVID-19 infection, antibodies in most serum samples were found to react strongly with coronaviruses OC43, HKU1, NL63 and 229E S1 antigens (Fig. 3c for COVID-19 patients and Fig. $3 \mathrm{~d}$ for pre-pandemic samples), consistent with the fact that most adults have been infected by common colds. Many of the common-cold antibodies showed high avidity ( $>0.5$, Fig. $4 d)$, suggesting old infections.

In pre-pandemic serum samples, antibodies against the common colds did not cross-react with SARS-CoV-2 or SARS, except for one sample showing detectable IgG against SARS S1 (Fig. 3d), because none were expected to be infected by SARS-CoV-2 or SARS (the latter infected $<9,000$ people worldwide and ended circula- 

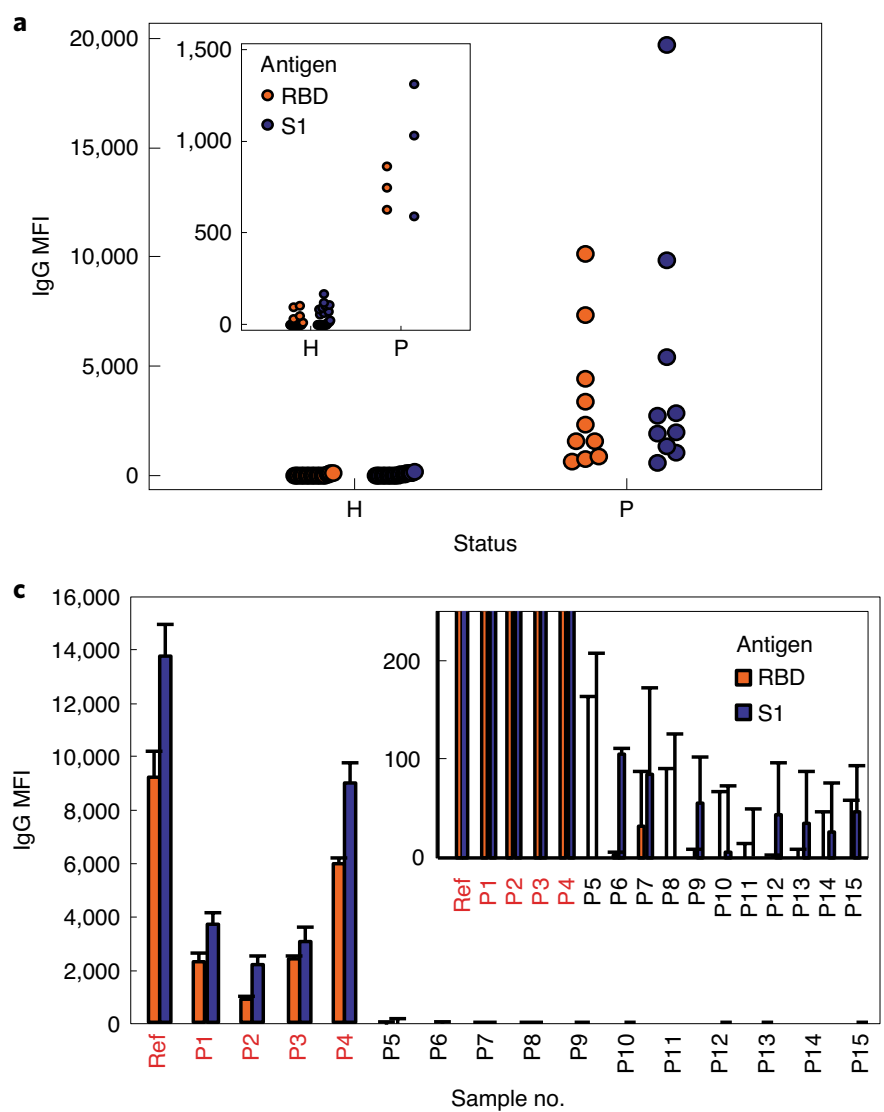

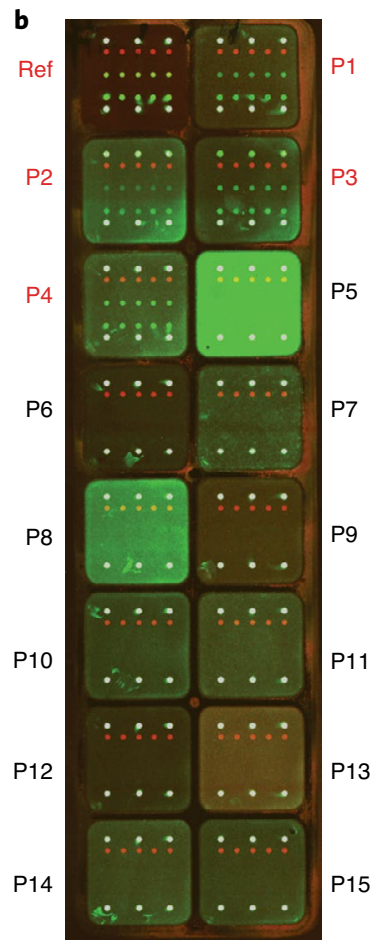

Fig. 5 | Detection of SARS-CoV-2 antibodies in human saliva. a, Distribution of IgG MFI signals from the saliva of COVID-19-positive patients ('P', $n=10$ ) compared to healthy controls (' $\mathrm{H}^{\prime}, n=23$ ) against both $\mathrm{S} 1$ and RBD, with the inset showing a noticeable difference between the weak IgG signals from COVID-19 saliva and signal noise from that of healthy saliva. $\mathbf{b}$, A confocal fluorescence image of IgG signals in the saliva of patients who had recovered from COVID-19 ( $n=4$, denoted P1-P4, in red) and healthy controls ( $n=11$, denoted P5-P15) and 10 4 times diluted serum of a patient with PCR-confirmed COVID-19 as a reference (denoted Ref, in red). The human IgG control spots are located on rows 1 and 7 , intrawell signal normalizer spots are on row 2 , RBD spots are on row 4, and S1 spots are on row 6 in each microarray. c, MFI signals of anti-S1 and anti-RBD IgG measured in saliva samples (P1-P15) and a PCR-positive COVID-19 serum reference (Ref) from the biochip seen in $\mathbf{b}$, with background signals subtracted. The MFI of healthy controls was near background noise, as shown in the inset. Error bars indicate one standard deviation from the mean of the MFI signals detected against the respective microarrayed antigen spots $(n=3)$. The raw data corresponding to $\mathbf{b}$ and $\mathbf{c}$ are provided in Supplementary Table 9.

tion in 2003). By contrast, we found that antibodies in SARS-CoV2 -infected patients showed obvious cross-reactivity to SARS S1 and RBD antigens (Fig. 3c), as also reported recently by others ${ }^{38,44}$ and attributed to a high degree $(>50 \%)$ of conservation of the amino-acid sequence between SARS-CoV-2 and SARS. However, owing to the long disappearance of SARS, the likelihood of false positives of COVID-19 serology caused by cross-reactivity to past SARS infection was low.

We observed that the avidity of the cross-reacting antibodies in COVID-19 sera against SARS was mostly low, with several noticeable exceptions of avidity approaching 0.5 (Fig. 4d), in contrast to the low avidity against SARS-CoV-2 (avidity indices $<0.2$ for the sample set 1 COVID-19 cohort). The observed high avidity of SARS-CoV-2 antibody cross-reacting with the SARS antigen was surprising, as the patients were not likely to have been infected by SARS previously, and could be a result of cross-reactivity with old infections by common human coronaviruses. This result is not understood and underscores the importance of further investigating the immune responses of SARS-CoV-2-infected patients in relation to the history of infections by previous same-family coronaviruses.

Detection of SARS-CoV-2 antibodies in human saliva. Finally, we exploited the high analytical sensitivity of the nanoplasmonic gold substrate for detecting antibodies in human saliva against SARS-CoV-2. It is well known that the antibody concentration in human saliva is orders of magnitude lower than in blood or serum, demanding assays with exquisite analytical sensitivity and capable of detecting high signal over background noise ${ }^{34}$. We first tested the saliva samples of 10 fully recovered individuals with PCR-confirmed COVID-19 (infection occurred more than approximately three to four months before saliva collection) and 23 healthy non-infected individuals on pGOLD. Although background fluorescence signals were observed for some saliva samples (Fig. 5b), probably due to the autofluorescence of molecules in the saliva, background-subtracted IgG signals on S1 and RBD antigens allowed clear differentiation of positive COVID-19-recovered saliva from healthy controls (Fig. $5 \mathrm{a}, \mathrm{c})$. Note that a COVID-19 serum sample diluted $10^{4}$ times was included in the assay (labelled 'Ref' in Fig. 5b,c) and showed a similar IgG level as in the COVID-19-positive saliva samples.

Next, we tested saliva samples collected from 14 patients with PCR-confirmed COVID-19, collected within 22 days post symptom onset. We detected IgG against the S1 and RBD antigen in 8 out of 10 saliva samples from patients infected $>10$ days prior to saliva collection (Supplementary Table 10). A substantial percentage of saliva from the recently infected patients also showed positive for $\mathrm{IgM}$, in contrast to that of fully recovered patients tested previously; 
however, a more systematic study is needed to discern IgM and IgG trends using saliva. Nevertheless, the results suggest the potential of antibody testing on pGOLD through non-invasive sample matrices such as saliva, which could greatly facilitate COVID-19 surveillance and prevalence studies.

\section{Discussion}

The pGOLD SARS-CoV-2 IgG/IgM assay has near $100 \%$ sensitivity for COVID-19 sera collected $>14$ days post infection and 99.78\% specificity in detecting both IgG and IgM subtypes against SARS-CoV-2 S1 and RBD antigens, based on 500 presumptive negative and positive samples (Supplementary Tables 11 and 12). The pGOLD SARS-CoV-2 IgG/IgM assay detected classical serological immune response behaviour of an earlier appearance of IgM than IgG post infection in the acute phase (0-14 days). Notably, $\sim 87 \%$ of patients developed detectable IgM antibodies $\geq 6$ days post symptom onset, which is substantial and could be a meaningful parameter to aid clinical decisions. The S1 subunit is a highly specific antigen of SARS-CoV-2, with the amino-acid sequence of the SARS-CoV-2 S1 subunit being only $25 \%$ or less conserved from four common human coronaviruses ${ }^{14}$. Consequently, the common-cold antibodies do not cross-react with SARS-CoV-2 S1 and RBD antigens in the pGOLD assay. The pGOLD assay is multiplexed, detecting IgM and IgG antibodies against multiple SARS-CoV-2 antigens simultaneously. It can also detect antibodies against SARS and common human coronaviruses in a single test run (Figs. 3 and 4).

Antibodies developed shortly after a primary infection exhibit low avidity and bind weakly to the antigen. Over time, avidity towards antigens can increase as antibodies 'mature' through colonial expansion, hypermutation and affinity selection in the germinal centre ${ }^{45}$. IgG avidity has been used previously to aid the differentiation of recent from past infection and distinguish primary from secondary infection ${ }^{25-29}$. Antibody avidity could also be useful in assessing vaccination efficacy ${ }^{46,47}$, immunity and convalescent plasma-based antibody therapy ${ }^{48}$.

In the current case, testing of a large number of samples from patients recovered from COVID-19 over long periods of time ( $>6$ months to years) is needed to glean a full picture of antibody maturation. The SARS-CoV-2 antibody-avidity index is expected to increase due to its similarity to SARS ${ }^{24,49}$, for which significant increase in antibody avidity over time (more than about six months, up to 269 days $)^{49}$ was observed. The same phenomenon was also noticed for the four common human coronaviruses OC43, HKU1, NL63 and 229E, for which the pGOLD assay indeed detected high antibody avidity, given that most adults have had common colds in the past. Virtually every adult serum sample suggested infection by at least one common cold with antibody avidity well above 0.5 (Fig. 4c,d). In addition to assessing recent versus old infection, multiplexed measurements of avidity towards a panel of antigens are useful for investigating immune responses to SARS-CoV-2, cross-reactivity due to past infections by viruses in the same family, and antibody maturation patterns against different viral targets.

We found that patients with COVID-19 showed low antibody avidity towards SARS-CoV-2. Because SARS-CoV-2 is a completely new virus, the lack of any immune memory is expected and consistent with the low avidity measured. High antibody avidity against SARS was observed with several patients newly infected by SARS-CoV-2, suggesting a degree of immune memory of old coronaviruses and warranting further investigations.

The pGOLD avidity measurement was useful for shedding light on the unusual immune responses of the PAMF-065 patient. The positive IgG against SARS-CoV-2 S1 (but not RBD) on day 6 post symptom onset with a high avidity of $>0.5$ suggested an anamnestic immune response caused by memory effects of past infection by related viruses. The patient was a 73-year-old woman who tested positive for COVID-19 by PCR at six days post symp- tom onset, including fever and lymphopenia. Notably, the patient did not develop pneumonia or get much sicker, despite her relatively advanced age, suggesting a degree of immunity developed due to a past infection. This case could be related to the so-called 'cross-immunity' between respiratory coronaviruses ${ }^{50}$.

Outlook. With $100 \%$ sensitivity two weeks post COVID-19 symptom onset and $99.78 \%$ specificity based on 454 negative samples, the pGOLD SARS-CoV-2 IgG/IgM assay is promising for population-based mass screening, as well as sero-surveillance and prevalence studies. The clear trend of IgM detection prior to IgG and $\sim 87 \%$ IgM positivitiy $\geq 6$ days post symptom onset could be used to assess responses to coronavirus infection starting from the early stage. Multiplexed antibody-avidity measurements against multiple viral antigens could facilitate our understanding of the immune responses and antibody maturation, reveal infection timing ${ }^{51}$ and decipher cross-reactivity with other common human coronaviruses. Just as important, the high sensitivity of SARS-CoV-2 antibody detection in human saliva samples on the nanotechnology-based pGOLD substrate could enable non-invasive sample collection at home for the screening of COVID-19.

\section{Methods}

Biological samples and materials. Seventy-four PCR-confirmed COVID-19 serum samples were provided by the California Department of Public Health $(\mathrm{CDPH})$ and the Dr Jack S. Remington Laboratory for Specialty Diagnostics (JSRLSD) at the Palo Alto Medical Foundation (PAMF). These samples were provided with information on the number of days between symptom onset and sample collection, excluding four (sample set 2). Another set of PCR-confirmed COVID-19 sera (with no known information on the number of days between symptom onset and sample collection) were obtained from Baptist Health South Florida and Loma Linda Medical Center (sample set 1). Thirty-three COVID-19 PCR-negative samples were provided by CDPH and Loma Linda Medical Center. A total of 311 pre-pandemic serum samples collected in 2017-2019 were obtained from JSRLSD, and 40 healthy control samples were acquired from the Arizona State University Health Services for projects before the SARS-CoV-2 outbreak. Seventy-three samples from patients with various diseases for cross-reactivity checking were provided by Loma Linda Medical Center, CDPH, Valley Medical Center in San Jose and JSRLSD or purchased commercially.

Saliva samples were collected through a simple method involving spitting into a plastic tube from 23 healthy donors, 10 patients fully recovered from COVID-19 who tested positive by PCR about three to four months before saliva collection, and 14 patients with PCR-confirmed COVID-19 (from the Sinai Hospital of Baltimore/ LifeBridge Health) within 22 days post symptom onset. Patient enrolment was performed in accordance with standard ethical principles and approved by the Institutional Review Board at Lifebridge Health in Baltimore. Written consent was obtained from all patients.

Saliva was immediately heated at $56^{\circ} \mathrm{C}$ for $30-45 \mathrm{~min}$ post collection to inactivate viruses and then frozen at $-20^{\circ} \mathrm{C}$ before shipping overnight to Nirmidas Biotech Inc. for testing. The saliva collection, inactivation and transport processes were simple and required no special stabilizing treatment. Saliva samples were centrifuged to remove any aggregates, and the supernatant was tested on the pGOLD assay. All samples used were de-identified.

We conjugated IRDye800 CW NHS ester (LI-COR Biosciences) and CF647 NHS ester (Millipore Sigma, SCJ4600048) to anti-human IgG and anti-human IgM, respectively. The IRDye800-labelled anti-human IgG and CF647-labelled anti-human IgM were used for two-colour simultaneous detection of IgG and IgM against $\mathrm{S} 1$ and RBD antigens on pGOLD.

Multiplexed pGOLD microarray printing. Each pGOLD slide (Nirmidas Biotech) was printed with two SARS-CoV-2 antigens S1 and RBD using a GeSiM Nano-Plotter 2.1 at the following concentrations: $60 \mu \mathrm{g} \mathrm{ml}^{-1}$ for SARS-CoV-2 S1 (40591-V08H, Sino Biological), $25 \mu \mathrm{g} \mathrm{ml}^{-1}$ for SARS-CoV-2 RBD (40592-V08H, Sino Biological), $43 \mu \mathrm{g} \mathrm{ml}^{-1}$ for SARS-CoV-1 S1 (40150-V08B1, Sino Biological), $25 \mu \mathrm{g} \mathrm{ml}^{-1}$ for SARS-CoV-1 RBD (40150-V08B2, Sino Biological), $25 \mu \mathrm{g} \mathrm{ml}^{-1}$ for HKU1 S1 (40602-V08H, Sino Biological), $26 \mu \mathrm{g} \mathrm{ml}^{-1}$ for HCoV-OC43 S1 + S2 ECD (40607-V08B, Sino Biological), $31.5 \mu \mathrm{g} \mathrm{ml}^{-1}$ for HCoV-NL63 S1 (40600-V08H, Sino Biological) and $26 \mu \mathrm{g} \mathrm{ml}^{-1}$ for HCoV-229E S1 (40601-V08H, Sino Biological). On the same biochip, $7.5 \mu \mathrm{g} \mathrm{ml}^{-1}$ human IgG and $50 \mu \mathrm{g} \mathrm{ml}^{-1}$ biotinylated bovine serum albumin (BSA-biotin, Thermo Fisher Scientific) were also printed to serve as a printing control and 'intrawell signal normalizer', respectively. The antigens were printed in quintuplicate for capturing antibodies in serum, plasma, whole blood or saliva (the current work focused on serum and saliva). Identical microarrays were printed on 16 isolated wells in each pGOLD biochip with a total of four biochips, resembling a 64-well plate using the FAST frame incubation chamber (Millipore 
Sigma). The antigen-printed biochips were vacuum-sealed and stored at $-80^{\circ} \mathrm{C}$ until use.

Multiplexed pGOLD assay procedure. Serum samples were heated at $56^{\circ} \mathrm{C}$ for $30 \mathrm{~min}$ to inactivate and reduce potential risk from any residual virus ${ }^{52-54}$ The heat-inactivated serum samples were immediately used or stored at $-80^{\circ} \mathrm{C}$ for later use. The pGOLD antibody assays were performed in a 16-well format in the following steps: (1) blocking-all wells were blocked with a blocking buffer including 1\% BSA in phosphate-buffered saline (PBS) for $30 \mathrm{~min}$ at room temperature; (2) sample incubation-each well was then incubated with $100 \mu \mathrm{l}$ of $200 \times$ diluted patient serum in fetal bovine serum (FBS) including $0.05 \%$ Tween 20 (FBST) or saliva ( $2 \times$ dilution) for $60 \mathrm{~min}$ at room temperature (a positive control (diluted patient serum) and blank control (FBST only) were also included in each biochip); (3) secondary antibody incubation-each well was subsequently incubated with a mixture of $4 \mathrm{nM}$ IRDye800-labelled anti-human IgG secondary antibody, $4 \mathrm{nM}$ CF647-labelled anti-human IgM secondary antibody and $6 \mathrm{nM}$ CF647-labelled streptavidin in 20\% FBS solution for $30 \mathrm{~min}$ at room temperature (Fig. 1a). Each well was washed six times with $1 \times$ PBST $(10 \times$ PBST: $10 \times$ PBS with $0.5 \%$ Tween 20) between steps. The CF647-labelled streptavidin in the detection step binds to BSA-biotin spots in each well on the pGOLD biochip, and the signal was used as an 'intrawell signal normalizer'; that is, the IgG and IgM signals were divided by the intrawell normalizing signal to obtain a ratio value for IgG and IgM of each sample, and then multiplied by 10 and 100, respectively (defined as the normalized IgG and IgM levels, respectively, throughout this work). Intrawell normalization was designed to minimize the effect of slight differences in pGOLD film uniformity across each biochip, which may affect fluorescence enhancement. Scaling by 10 or 100 was intended to make cutoff values $>1$ and avoid small decimal numbers.

pGOLD coronavirus avidity assay. The pGOLD IgG avidity in a serum sample was measured by detecting captured IgG for the sample with and without urea treatment side-by-side in two neighbouring pGOLD wells. In each well, $0.5 \mu \mathrm{l}$ of the same serum sample was diluted 200 times. In one well, a regular IgG assay was performed, whereas in the neighbouring well the same IgG assay was performed except that a 10 -min treatment with $6 \mathrm{M}$ urea was added following the sample incubation step. Such treatment by a denaturing agent like urea could detach the IgG from the antigen spot if the IgG avidity is low. At the end of the assay, the IgG signal of the urea-treated sample was divided by the IgG signal of the regularly assayed sample, giving a normalized avidity value.

Data analysis. After the assay procedures, a dual-channel MidaScan microarray scanner (Nirmidas Biotech) was used to scan each biochip for IRDye800-conjugated anti-human IgG and CF647-conjugated anti-human IgM signals on the antigen spots. CF647 and IRDye800 fluorescence images in the respective red and green channels were generated and the MFI signal for each microarray was quantified by MidaScan Software version 2.0.0. The data were used to calculate the average MFI, with the antigen spots of the highest and lowest MFIs removed for each channel, thus leading to a single signal intensity used to measure antibody detection in each sample. Afterwards, the MFI was divided by the average intrawell MFI signal, resulting in an intrawell ratio, and scaled by a factor of 100 for IgM and 10 for IgG. The final antibody level values were used to determine the antibody status of the samples for the corresponding antigen. Cutoffs were determined by ROC curve analysis using MedCalc Statistical Software version 19.2.1 (MedCalc Software). This method resulted in the optimal combination of sensitivity and specificity of the multiplexed assay on pGOLD.

Reporting summary. Further information on research design is available in the Nature Research Reporting Summary linked to this Article.

\section{Data availability}

The main data supporting the results in this study are available within the paper and its Supplementary Information. Raw datasets are available in figshare with the identifier https://doi.org/10.6084/m9.figshare.13011527 (ref. ${ }^{55}$ ).

Received: 8 July 2020; Accepted: 5 October 2020;

Published online: 29 October 2020

\section{References}

1. Corman, V. M. et al. Detection of 2019 novel coronavirus (2019-nCoV) by real-time RT-PCR. Eurosurveillance https://doi.org/10.2807/1560-7917. ES.2020.25.3.2000045 (2020).

2. Tahamtan, A. \& Ardebili, A. Real-time RT-PCR in COVID-19 detection: issues affecting the results. Expert Rev. Mol. Diagn. 20, 453-454 (2020).

3. Kashir, J. \& Yaqinuddin, A. Loop mediated isothermal amplification (LAMP) assays as a rapid diagnostic for COVID-19. Med. Hypotheses https://doi. org/10.1016/j.mehy.2020.109786 (2020).

4. Yu, L. et al. Rapid detection of COVID-19 coronavirus using a reverse transcriptional loop-mediated isothermal amplification (RT-LAMP) diagnostic platform. Clin. Chem. 66, 975-977 (2020).
5. Petherick, A. Developing antibody tests for SARS-CoV-2. Lancet 395 1101-1102 (2020).

6. Vashist, S. K. In vitro diagnostic assays for COVID-19: recent advances and emerging trends. Diagnostics https://doi.org/10.3390/diagnostics 10040202 (2020)

7. Younes, N. et al. Challenges in laboratory diagnosis of the novel coronavirus SARS-CoV-2. Viruses https://doi.org/10.3390/v12060582 (2020).

8. Montesinos, I. et al. Evaluation of two automated and three rapid lateral flow immunoassays for the detection of anti-SARS-CoV-2 antibodies. J. Clin. Virol. https://doi.org/10.1016/j.jcv.2020.104413 (2020).

9. Tre-Hardy, M. et al. Validation of a chemiluminescent assay for specific SARS-CoV-2 antibody. Clin. Chem. Lab. Med. 58, 1357-1364 (2020).

10. Amanat, F. et al. A serological assay to detect SARS-CoV-2 seroconversion in humans. Nat. Med. 26, 1033-1036 (2020).

11. Lassaunière, R. et al. Evaluation of nine commercial SARS-CoV-2 immunoassays. Preprint at medRxiv https://doi. org/10.1101/2020.04.09.20056325 (2020).

12. Adams, E. R. et al. Antibody testing for COVID-19: a report from the National COVID Scientific Advisory Panel. Wellcome Open Res 5, 139 (2020).

13. Zhao, J. et al. Antibody responses to SARS-CoV-2 in patients of novel coronavirus disease 2019. Clin. Infect. Dis. https://doi.org/10.1093/cid/ciaa344 (2020).

14. Okba, N. M. A. et al. Severe acute respiratory syndrome coronavirus 2 -specific antibody responses in coronavirus disease 2019 patients. Emerg. Infect. Dis. 26, 1478-1488 (2020).

15. Infantino, M. et al. Diagnostic accuracy of an automated chemiluminescent immunoassay for anti-SARS-CoV-2 IgM and IgG antibodies: an Italian experience. J. Med. Virol. 92, 1671-1675 (2020).

16. Theel, E. S., Harring, J., Hilgart, H. \& Granger, D. Performance characteristics of four high-throughput immunoassays for detection of IgG antibodies against SARS-CoV-2. J. Clin. Microbiol. https://doi.org/10.1128/jcm.01243-20 (2020).

17. Tang, M. S. et al. Clinical performance of the Roche SARS-CoV-2 serologic assay. Clin. Chem. 66, 1107-1109 (2020).

18. Bendavid, E. et al. COVID-19 antibody seroprevalence in Santa Clara County, California. Preprint at medRxiv https://doi.org/10.1101/2020.04.14.20062463 (2020).

19. Xu, X. et al. Seroprevalence of immunoglobulin $M$ and $G$ antibodies against SARS-CoV-2 in China. Nat. Med. 26, 1193-1195 (2020).

20. Reifer, J., Hayum, N., Heszkel, B., Klagsbald, I. \& Streva, V. A. SARS-CoV-2 IgG antibody responses in New York City. Diagn. Microbiol. Infect. Dis. https://doi.org/10.1016/j.diagmicrobio.2020.115128 (2020).

21. Sood, N. et al. Seroprevalence of SARS-CoV-2-specific antibodies among adults in Los Angeles County, California, on April 10-11, 2020. JAMA 323, 2425-2427 (2020).

22. Levesque, J. \& Maybury, D. W. A note on COVID-19 seroprevalence studies: a meta-analysis using hierarchical modelling. Preprint at medRxiv https://doi org/10.1101/2020.05.03.20089201 (2020).

23. Tang, M. S. et al. Clinical performance of two SARS-CoV-2 serologic assays. Clin. Chem. 66, 1055-1062 (2020).

24. Bauer, G. The variability of the serological response to SARS-corona virus-2: potential resolution of ambiguity through determination of avidity (functional affinity). J. Med. Virol. https://doi.org/10.1002/jmv.26262 (2020).

25. Gutierrez, J. \& Maroto, C. Are IgG antibody avidity assays useful in the diagnosis of infectious diseases? A review. Microbios 87, 113-121 (1996).

26. Suligoi, B. et al. Precision and accuracy of a procedure for detecting recent human immunodeficiency virus infections by calculating the antibody avidity index by an automated immunoassay-based method. J. Clin. Microbiol. 40, 4015-4020 (2002)

27. Revello, M. G. et al. Diagnosis and outcome of preconceptional and periconceptional primary human cytomegalovirus infections. J. Infect. Dis. 186, 553-557 (2002)

28. De Souza, V. A. U. F. et al. Use of an immunoglobulin G avidity test to discriminate between primary and secondary Dengue virus infections. J. Clin. Microbiol. 42, 1782-1784 (2004).

29. Zhang, B. et al. Diagnosis of Zika virus infection on a nanotechnology platform. Nat. Med. 23, 548-550 (2017).

30. Hedman, K. \& Seppala, I. Recent rubella virus infection indicated by a low avidity of specific IgG. J. Clin. Immunol. 8, 214-221 (1988)

31. Tabakman, S. M. et al. Plasmonic substrates for multiplexed protein microarrays with femtomolar sensitivity and broad dynamic range. Nat. Commun. 2, 466 (2011).

32. Zhang, B., Kumar, R. B., Dai, H. \& Feldman, B. J. A plasmonic chip for biomarker discovery and diagnosis of type 1 diabetes. Nat. Med. 20, 948-953 (2014)

33. Li, X. et al. Multiplexed anti-toxoplasma IgG, IgM and IgA assay on plasmonic gold chips: towards making mass screening possible with dye test precision. J. Clin. Microbiol. 54, 1726-1733 (2016). 
34. Li, X. et al. Plasmonic gold chips for the diagnosis of Toxoplasma gondii, CMV and rubella infections using saliva with serum detection precision. Eur. J. Clin. Microbiol. Infect. Dis. 38, 883-890 (2019).

35. Chen, $X$. et al. A novel quantitative microarray antibody capture assay identifies an extremely high hepatitis delta virus prevalence among hepatitis B virus-infected Mongolians. Hepatology 66, 1739-1749 (2017).

36. Walls, A. C. et al. Structure, function and antigenicity of the SARS-CoV-2 spike glycoprotein. Cell 181, 281-292 (2020).

37. Liu, W. et al. Evaluation of nucleocapsid and spike protein-based enzyme-linked immunosorbent assays for detecting antibodies against SARS-CoV-2. J. Clin. Microbiol. https://doi.org/10.1128/jcm.00461-20 (2020).

38. Long, Q.-X. et al. Antibody responses to SARS-CoV-2 in patients with COVID-19. Nat. Med. 26, 845-848 (2020).

39. Yan, R. et al. Structural basis for the recognition of SARS-CoV-2 by full-length human ACE2. Science 367, 1444-1448 (2020).

40. Wu, Y. et al. Identification of human single-domain antibodies against SARS-CoV-2. Cell Host Microbe 27, 891-898 (2020).

41. Yuan, M. et al. A highly conserved cryptic epitope in the receptor binding domains of SARS-CoV-2 and SARS-CoV. Science 368, 630-633 (2020).

42. Prince, H. E., Yeh, C. \& Lapé-Nixon, M. Utility of IgM/IgG ratio and IgG avidity for distinguishing primary and secondary Dengue virus infections using sera collected more than 30 days after disease onset. Clin. Vaccin. Immunol. 18, 1951-1956 (2011).

43. Pinto, D. et al. Cross-neutralization of SARS-CoV-2 by a human monoclonal SARS-CoV antibody. Nature 583, 290-295 (2020).

44. Chia, W. N. et al. Serological differentiation between COVID-19 and SARS infections. Emerg. Microbes Infect. 9, 1497-1505 (2020).

45. Victora, G. D. \& Nussenzweig, M. C. Germinal centers. Annu. Rev. Immunol. 30, 429-457 (2012).

46. Usinger, W. R. \& Lucas, A. H. Avidity as a determinant of the protective efficacy of human antibodies to pneumococcal capsular polysaccharides. Infect. Immun. 67, 2366-2370 (1999).

47. Dobaño, C. et al. Concentration and avidity of antibodies to different circumsporozoite epitopes correlate with RTS,S/AS01E malaria vaccine efficacy. Nat. Commun. 10, 2174 (2019).

48. Sheridan, C. Convalescent serum lines up as first-choice treatment for coronavirus. Nat. Biotechnol. 38, 655-658 (2020).

49. Chan, K. H. et al. Use of antibody avidity assays for diagnosis of severe acute respiratory syndrome coronavirus infection. Clin. Vaccin. Immunol. 14, 1433-1436 (2007)

50. Yaqinuddin, A. Cross-immunity between respiratory coronaviruses may limit COVID-19 fatalities. Med. Hypotheses https://doi.org/10.1016/j. mehy.2020.110049 (2020).

51. Furuya, A. K. M. et al. Use of the immunoglobulin $G$ avidity assay to differentiate between recent Zika and past Dengue virus infections. Clin. Sci. 133, 859-867 (2019).
52. Burbelo, P. D. et al. Sensitivity in detection of antibodies to nucleocapsid and spike proteins of severe acute respiratory syndrome coronavirus 2 in patients with coronavirus disease 2019. J. Infect. Dis. 222, 206-213 (2020).

53. Batéjat, C., Grassin, Q., Manuguerra, J.-C. \& Leclercq, I. Heat inactivation of the severe acute respiratory syndrome coronavirus 2. Preprint at bioRxiv https://doi.org/10.1101/2020.05.01.067769 (2020).

54. Wang, T., Lien, C., Liu, S. \& Selveraj, P. Effective heat inactivation of SARS-CoV-2. Preprint at medXriv https://doi. org/10.1101/2020.04.29.20085498 (2020).

55. Liu, T. et al. Dataset for quantification of antibody avidities and accurate detection of SARS-CoV-2 antibodies in serum and saliva on plasmonic substrates (Figshare, 2020); https://doi.org/10.6084/m9.figshare.13011527

\section{Acknowledgements}

We thank L. Drake of the Loma Linda Medical Center for providing some of the samples used in this work, and W. Li and J. Sui for providing purified humanized anti-SARS-CoV-2 IgG and for helpful discussions.

\section{Author contributions}

H.D. and M.T. envisioned and designed the project and experiments. T.L., J.H., S.Z., J.K. and D.S. performed the assay experiments. K.O., D.K., S.T.C., J.R., C.V.H., C.P., J.B., J.P.R.-S., J.G.M., K.P.B., P.A.G. and U.S.T. collected and processed samples. Co-authors from PAMF worked on sample collection and accessing patient information through an IRB (protocols 1588570-3 and 830133-7). Co-authors at Sinai Hospital of Baltimore contributed through the study 'The Evaluation of Hemostasis by Thromboelastography, Platelet Function Testing, and Biomarker Analysis in Hospitalized COVID-19 Patients (TARGET-COVID Study)' (ClinicalTrials.gov identifier NCT04493307). H.D., J.H. and T.L. analysed the data and wrote the manuscript. All authors commented on the manuscript.

\section{Competing interests}

H.D. worked on this project as a consultant for Nirmidas Biotech, independently of Stanford Projects. The remaining authors declare no competing interests.

\section{Additional information}

Supplementary information is available for this paper at https://doi.org/10.1038/ s41551-020-00642-4.

Correspondence and requests for materials should be addressed to J.G.M., M.T. or H.D. Reprints and permissions information is available at www.nature.com/reprints. Publisher's note Springer Nature remains neutral with regard to jurisdictional claims in published maps and institutional affiliations.

(c) The Author(s), under exclusive licence to Springer Nature Limited 2020 


\section{Reporting Summary}

Nature Research wishes to improve the reproducibility of the work that we publish. This form provides structure for consistency and transparency in reporting. For further information on Nature Research policies, see our Editorial Policies and the Editorial Policy Checklist.

\section{Statistics}

For all statistical analyses, confirm that the following items are present in the figure legend, table legend, main text, or Methods section.

n/a Confirmed

\The exact sample size $(n)$ for each experimental group/condition, given as a discrete number and unit of measurement

$\bigotimes$ A statement on whether measurements were taken from distinct samples or whether the same sample was measured repeatedly

$\chi$ The statistical test(s) used AND whether they are one- or two-sided

Only common tests should be described solely by name; describe more complex techniques in the Methods section.

$\bigotimes \square$ A description of all covariates tested

Х $\square$ A description of any assumptions or corrections, such as tests of normality and adjustment for multiple comparisons

$\square$ A full description of the statistical parameters including central tendency (e.g. means) or other basic estimates (e.g. regression coefficient)

A full description of the statistical parameters including central tendency (e.g. means) or other basic esti
AND variation (e.g. standard deviation) or associated estimates of uncertainty (e.g. confidence intervals)

$X \square$ For null hypothesis testing, the test statistic (e.g. $F, t, r$ ) with confidence intervals, effect sizes, degrees of freedom and $P$ value noted

Give $P$ values as exact values whenever suitable.

Х $\square$ For Bayesian analysis, information on the choice of priors and Markov chain Monte Carlo settings

$\square$ ×or hierarchical and complex designs, identification of the appropriate level for tests and full reporting of outcomes

Х $\square$ Estimates of effect sizes (e.g. Cohen's $d$, Pearson's $r$ ), indicating how they were calculated

Our web collection on statistics for biologists contains articles on many of the points above.

\section{Software and code}

Policy information about availability of computer code

Data collection A dual-channel MidaScan microarray scanner (Nirmidas Biotech, Inc.) was used to scan each biochip for IRDye800-conjugated anti-human IgG and CF647-conjugated anti-human IgM signals on the SARS-CoV-2 antigen spots. CF647 and IRDye800 fluorescence images in the respective red and green channels were generated, and the median fluorescence signal (MFI) for each microarray was quantified by the MidaScan Software version 2.0.0. The data were used to calculate the average MFI with the antigen spots of the highest and lowest MFIs removed for each channel, thus leading to a single signal intensity used to measure antibody detection in each sample. Afterwards, the MFI was normalized to the average intrawell MFI signal, resulting in an intrawell ratio, and adjusted by a factor of 100 for IgM and 10 for IgG. The final data were exported to an Excel sheet.

Data analysis Histograms were created with Excel 365. The box-plot analysis and correlation analysis were done using MedCalc Statistical Software version 19.2.1 (MedCalc Software Ltd., Ostend, Belgium). Cutoffs were determined by ROC-curve analysis using MedCalc Statistical Software version 19.2.1. This method resulted in the optimal combination of sensitivity and specificity of the multiplexed assay on pGOLD. 
Policy information about availability of data

All manuscripts must include a data availability statement. This statement should provide the following information, where applicable:

- Accession codes, unique identifiers, or web links for publicly available datasets

- A list of figures that have associated raw data

- A description of any restrictions on data availability

The main data supporting the results in this study are available within the paper and its Supplementary Information. Raw datasets are available in figshare with the identifier doi:10.6084/m9.figshare.13011527.

\section{Field-specific reporting}

Please select the one below that is the best fit for your research. If you are not sure, read the appropriate sections before making your selection.

$\bigotimes$ Life sciences $\square$ Behavioural \& social sciences $\square$ Ecological, evolutionary \& environmental sciences

For a reference copy of the document with all sections, see nature.com/documents/nr-reporting-summary-flat.pdf

\section{Life sciences study design}

All studies must disclose on these points even when the disclosure is negative.

Sample size All samples that could be obtained during the start of the COVID-19 pandemic were included in the study.

593 serum samples:

74 SARS-CoV-2 PCR+ with disease onset data;

62 SARS-COV-2 PCR+ without disease onset data;

33 SARS-CoV-2 PCR-;

311 pre-pandemic samples;

73 other autoimmune or infectious diseases;

40 healthy control samples acquired before the SARS-CoV-2 outbreak.

35 Saliva samples:

10 fully recovered COVID-19 patients;

14 PCR confirmed COVID-19 patients;

11 healthy controls.

Data exclusions No data were excluded, but different subsets were used for different analyses.

For ROC-curve analysis and cutoff determination, we used samples from 62 patients (without disease onset data) and $384(33+311+40)$ negative samples.

For the disease onset-related sensitivity study, we used samples from 74 patients with disease-onset data.

For the avidity study, we used S1/RBD IgG positive samples from 74 patients with disease-onset data.

For the saliva test, we used 15 saliva samples with one serum sample as a positive control.

Replication The assays were repeated and performed by two independent researchers.

Randomization Randomization did not apply to this study, because the purpose of the work was assay development.

Blinding

The assay tests were performed with blinding. Data analyses were done with known disease status or onset days (and no other information).

\section{Reporting for specific materials, systems and methods}

We require information from authors about some types of materials, experimental systems and methods used in many studies. Here, indicate whether each material, system or method listed is relevant to your study. If you are not sure if a list item applies to your research, read the appropriate section before selecting a response. 


\begin{tabular}{l|l}
\hline$n / a$ & Involved in the study \\
\hline & $\bigotimes$ Antibodies \\
$\square$ & $\square$ Eukaryotic cell lines \\
$\square$ & $\square$ Palaeontology and archaeology \\
$\square$ & $\square$ Humals and other organisms \\
$\square$ & $\square$ Clinical data \\
$\searrow$ & $\square$ Dual use research of concern
\end{tabular}

\section{Antibodies}

Antibodies used

Validation
Goat anti-human IgG Fcy Fragment Specific from Jackson ImmunoResearch LABORATORIES, Inc. Code Number: 109-005-098. Goat anti-human IgM , Fc5 $\mu$ Fragment Specific Jackson ImmunoResearch LABORATORIES, Inc. Code Number: 109-005-129.

All the antibodies used in the pGOLD assay were tested and confirmed to specifically bind human IgG or IgM antibodies in our previous published work. The antibody specificity was also stated by Jackson ImmunoResearch (https://www.jacksonimmuno.com/ catalog/products/109-005-098; and https://www.jacksonimmuno.com/catalog/products/109-005-129).

\section{Human research participants}

Policy information about studies involving human research participants

Population characteristics

Recruitment

Ethics oversight
Only deidentified samples were used. 136 samples were from SARS-CoV-2 PCR+ COVID19 patients, 33 samples were from SARS-CoV-2 PCR-controls, 424 negative control samples were from a non-COVID19 infected diseases (pre-pandemic).

All serum samples were preexisting. For saliva samples, patients and healthy controls agreed to be enrolled. 